\title{
Pseudo resistivity section by VLF-EM method with a single frequency
}

\author{
Daisuke HYODO $^{1}$, Hitoshi MIKADA ${ }^{1}$, Tada-nori GOTO ${ }^{1}$ and Junichi TAKEKAWA ${ }^{1}$ \\ ${ }^{1}$ Dept. of Civil and Earth Res. Eng., Kyoto University
}

\begin{abstract}
Electromagnetic waves with single or limited frequencies from VLF transmitters generate secondary induced components of magnetic field, which are used to detect localized changes in electrical conductivity contrasts. This method, so-called VLF-EM, has been the powerful tool for mapping subsurface geological structures because of its low cost and short survey terms compared with electrical resistivity survey. However, it has not been tested to estimate a pseudo-resistivity section, both the apparent resistivity and the depth of conductive anomaly by using the measured magnetic components with a single frequency. In this study, the Normalized Full Gradient (NFG) method, generally used for the downward continuation of the potential filed data, was applied to the magnetic components at the surface. The VLF-EM data set was obtained numerically on a synthetic model. The cross section of NFG values derived from horizontal component of magnetic field clearly indicates high peaks at edges of a low resistivity anomaly buried below the surface. The peak of NFG values from the vertical component correspond with the centre of the anomaly. On the basis of the results, we estimated the pseudo-section of apparent resistivity from the VLF-EM data weighted with the NFG values at each depth. We confirmed that the weighted apparent resistivity values are lower in the vicinity of low resistivity anomaly than the surrounding area, although the estimated value is a little higher than the original value. We conclude that our simple technique give an approximate subsurface resistivity structures quickly, which is useful for geological interpretations.
\end{abstract}

\section{INTRODUCTION}

The electrical prospecting (DC resistivity survey) has been generally used to visualize near-surface resistivity structures (e.g., Zohby, 1969). However, to carry out this method, we have to put a lot of electrodes in the survey area and it is sometimes difficult to make sufficient investigations owing to the situation of geological features (e.g., dry surface condition) and the topography. While, the VLF-EM method can be carried out briefly, by using the electromagnetic wave from the powerful VLF stations situated at several locations around the world. Each VLF station always transmits the radio waves with a single frequency. When the wave arrive at subsurface resistivity anomalies -for example, aquifer, active faults and so on, both the vertical and the horizontal magnetic field due to electromagnetic induction are measured at the surface.

Recent studies about the VLF-EM methods allow us to estimate the apparent resistivity at a single frequency from the secondary vertical and horizontal magnetic field (Gharibi et al, 1999; Becken et al, 2003). Also, the discrete linear filtering technique (Fraser, 1969; Karous et al, 1983) is effective method to detect the depth of the anomalies. However, it was difficult to obtain both the information about the depth and the resistivity at the same time without using the complicated inversion techniques. To improve this problem and obtain the underground pseudo-section quickly, we try to estimate the section of the pseudo section of apparent resistivity by using the measured magnetic field.

\section{METHOD}

In this study, the VLF-EM data set was obtained numerically on a synthetic model. We compute the secondary vertical and horizontal magnetic field at the surface with a single frequency $(20 \mathrm{kHz})$ by using a three-dimensional finite difference algorithm. Then, we focus on the Normalized Full Gradient (NFG) method to obtain the depth information of resistivity anomaly. If the potential field satisfy Laplace's equation, the NFG method is generally used for the downward continuation of potential field data. Here, we try to apply the NFG method to the calculated magnetic field. The detail 
approach is as follwos. First, the the VLF data is convoluted with the function:

$$
w(y, z)=\int_{-\infty}^{\infty} e^{i w y+|\omega| z} d \omega
$$

The downward continuation $U$ of the measured potential $H$ is described as:

$$
U(y, z)=\int_{-\infty}^{\infty} H(\xi) w(y-\xi, z) d \xi
$$

To estimate the downward continuation, the fourier transform is applied. Then, inverse fourier transform with weighting estimates $U$. The NFG operator $\mathrm{G}$ is described as follows (e.g., Sindirgi et al., 2008):

$$
G\left(y_{i}, z_{j}\right)=\frac{\sqrt{\left(\frac{\partial U\left(y_{i}, z_{j}\right)}{\partial y}\right)^{2}+\left(\frac{\partial U\left(y_{i}, z_{j}\right)}{\partial z}\right)^{2}}}{\frac{1}{M} \sum_{i=1}^{M} \sqrt{\left(\frac{\partial U\left(y_{i}, z_{j}\right)}{\partial y}\right)^{2}+\left(\frac{\partial U\left(y_{i}, z_{j}\right)}{\partial z}\right)^{2}}} \text { (3) }
$$

where $M$ is the number of observation points, $\partial U / \partial y$ and $\partial U / \partial z$ are derivatives of the function $U$ with respect to $\mathrm{y}$ and $\mathrm{z}$-directions, respectively. It is important to determine the maximam limit of harmonic in downward continuation process (Dondurur, 2005; Sindirgi et al., 2008; Fedi et al, 2011). We examine the downward continuation field with the NFG operator, which give us better images of resistivity anomaly.

Our aim is not only estimation of depth of anomalies but also the evaluation of pseodo-section of apparent resistivity. As indicated in Gharibi and Pedersen (1999) by using the secondary vertical and horizontal magnetic field measured at the surface, we can compute the impedance and the apparent resistivity along the $y$-axis employing two following equations,

$$
\begin{gathered}
Z_{x y}(y)=Z_{x y}(0) \frac{H_{y}(0)}{H_{y}(y)}+\frac{\int_{0}^{y} i \omega \mu_{0} H_{z}\left(y_{0}\right) d y_{0}}{H_{y}(y)} \\
\rho_{a}(y)=\frac{1}{\mu_{0} \omega}\left|Z_{x y}(y)\right|^{2}
\end{gathered}
$$

where $\mu_{0}$ is the magnetic permiability of free air, and $\omega$ is angular frequency. The apparent resistivity in Eq (4) is for the surface. For estimation of apparent resistivity at a depth, we multiply the computed magnetic field by the NFG operator and substitute the new magnetic field for $\mathrm{Hy}$ and $\mathrm{Hz}$ in the Eqs.(2) and (3). Here, we call the cross section with the weighted apparent resistivity as "pseudo-resistivity section".
For numercial simulation, we assume the resistivity model as shown in Figure1. This model has background resistivity 1000 ohm-m. A resistivity anomaly of $100 \mathrm{ohm}-\mathrm{m}$ is embedded in a model with the width of $60 \mathrm{~m}$ and a thickness of $10 \mathrm{~m}$.

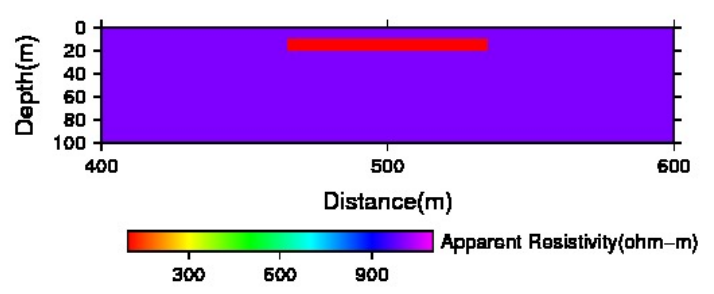

Figure 1 A model of subserface resistivity structure

\section{RESULTS AND DISCUSSIONS}

Figure 2 and 3 show the results of the NFG applying to $\mathrm{Hy}$ and $\mathrm{Hz}$ measured at the surface. It is confirmed that the location of the resistivity anomaly is detected by controlling the harmonic limits in the downward continuation process. Note that, the harmonic limits has a relationship with the resolution for the anomaly below the surface. If we choose the proper maximum limit of the wavelength similar to the anomaly's depth, we can detect the correct location of the resistivity anomaly. For example, in the case that the anomaly is buried at the deprh of $10 \mathrm{~m}, \mathrm{NFG}$ values and an proper high NFG area overlapping to the assumed anomaly is obtained if we choose the harmonic limit as 50 . Also, figure 2 shows that the NFG from the horizontal magnetic field can determine the both edges of the anomaly. Figure 3 shows that the NFG from the vertical magnetic field can detemine the center of the anomaly.

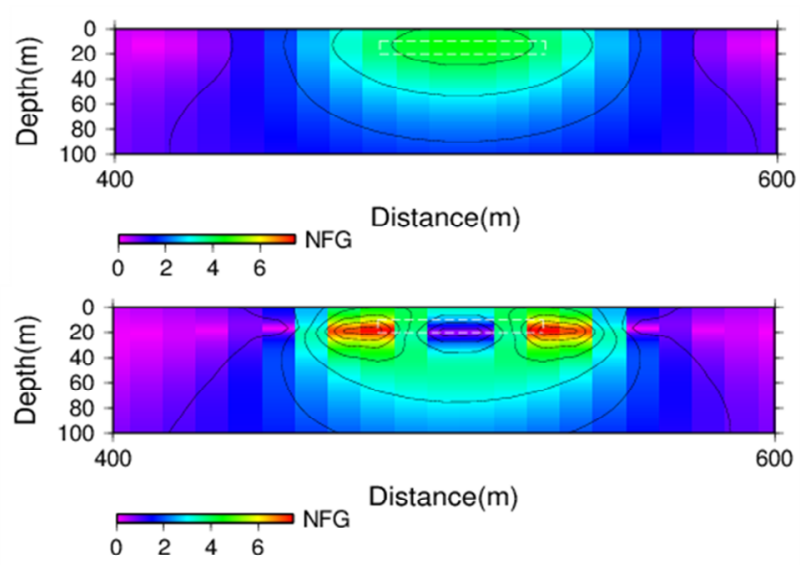

Figure 2 Cross section of the NFG applying to Hy (top: harmonic limit $=17$, bottom: harmonic limit $=$ 50). The bottom one show the clearer contour map, while the top one show ambiguous image judging from the NFG value and the contour lines. 


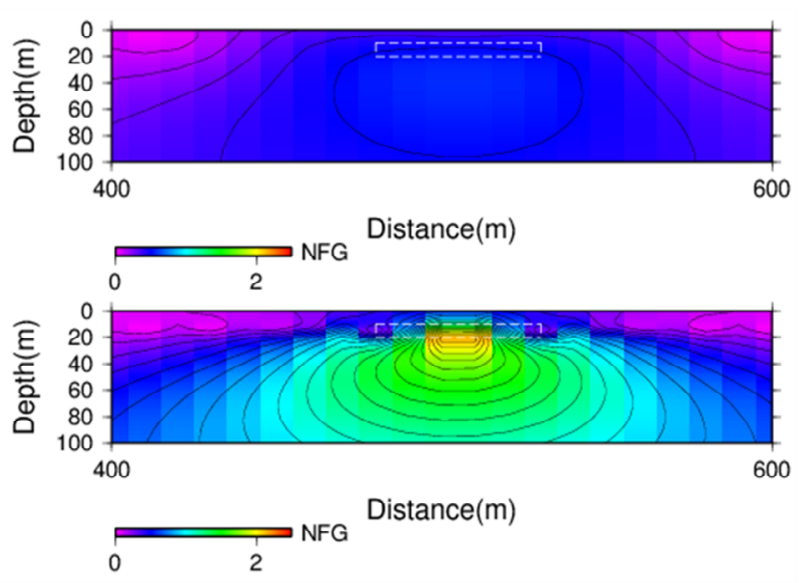

Figure 3 Cross section of the NFG applying to $\mathrm{Hz}$ (top: harmonic limit $=17$, bottom: harmonic limit $=$ 50). The bottom one show the clearer contour map, while the top one show ambiguous image judging from the NFG value and the contour lines.
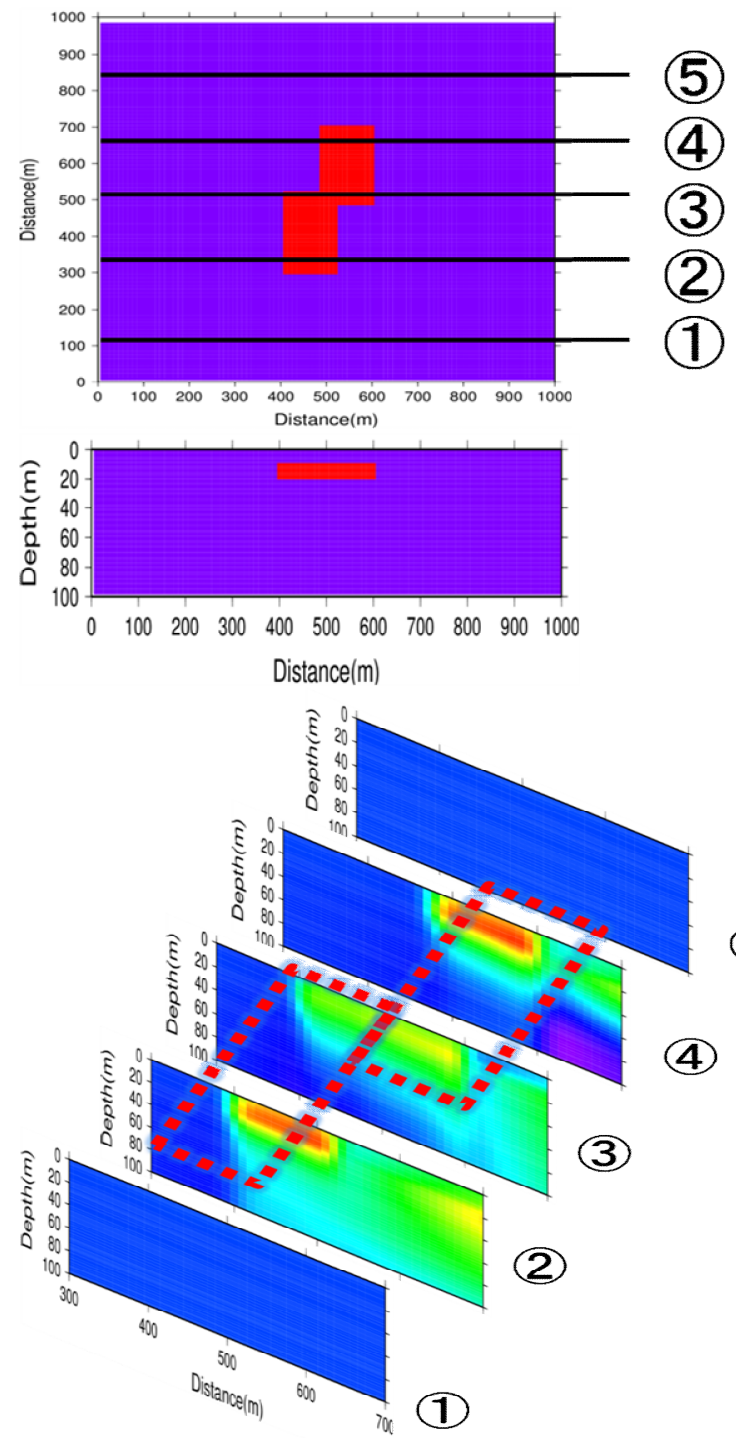

Figure 4 Top: A plane view of a 3D synthetic model and a cross section of line 3, Bottom: Estimated pseudo-resistivity sections of each line
We also obtained the pseudo-resistivity sections, similar to the assumed model. Figure 4 shows the estimated cross sections of apparent resistivity. It is confirmed that the estimated apparent resistivity is lower around the assumed anomaly. However, the higher apparent resistivity value is obtained at the centre of the resistivity anomaly, owing to the effect of the NFG value. Also, the background resistivity is imaged higher than the original one.

\section{CONCLUSION}

In this study, we tried to apply the NFG method to the VLF-EM data set and visualize the subsurface apparent resistivity distribution. We succeeded applying the NFG method to the VLF-EM data. In this method, it is possible to detect the both edges of the anomaly from horizontal magnetic components, and the center of the anomaly from the vertical magnetic components. Also, we show that pseudo-resistivity section estimated from the measured magnetic fields at the surface with a single frequency. Our brief way to obtain subsurface information will be useful for geological interpretation beacuse of its low cost and survey speed. The pseudo-resistivity section is also useful for an initial model for inversion of three-dimensional resistivity structure.

\section{REFERENCES}

1) Becken, M. and Pedersen, L. B., 2003, Transformation of VLF anomaly maps into apparent resistivity and phase, Geophysics, 68, 497-505

2) Fedi, M. and Florio, M., 2011, Normalized downward continuation of potential fields within the quasi-harmonic region, Geophysical Prospecting, 59, 1087-1100

3) Fraser, D.C., 1969, Contouring of VLF-EM data, Geophysics, 34, 958-967

4) Gharibi, M., and Pedersen, L. B., 1999, Transformation of VLF data into apparent resistivities and phases, Geophysics, 64, 1393-1402

5) Karous, M. and Hjelt, S.E., 1983, Linear Filtering of VLF dip-angle measurements, Geophysical Prospecting, 31, 782-794

6) Sindirgi, P., Pamukcu, Oya and Ozyalin, S, 2008, Application of Normalized Full Gradinet method to Self Potential (SP) data, Pure and Applied Geophysics, 409-427

7) Zohby, AA.R., 1969, The use of schulumberger and equational surroundings in groundwater investigation near el paso, texas, 1969, Geophysics, 34, 713-728 
